03

\title{
Электропроводность и диэлектрические характеристики твердых растворов $\mathrm{TI}\left(\mathrm{GaS}_{2}\right)_{1-x}(\operatorname{lnSe})_{x}$
}

\author{
(C) В.Г. Гуртовой ${ }^{1}$, А.У. Шелег ${ }^{1}$, С.Н. Мустафаева ${ }^{2}$, Э.М. Керимова ${ }^{2}$, С.Г. Джафрарова ${ }^{3}$ \\ ${ }^{1}$ НПЦ НАН Беларуси по материаловедению, \\ Минск, Беларусь \\ ${ }^{2}$ Институт фризики НАН Азербайджана, \\ Баку, Азербайджан \\ ${ }^{3}$ Гянджинский государственный университет, \\ Гянджа, Азербайджан \\ E-mail: hurtavy@physics.by
}

(Поступила в Редакцию 30 января 2017 г.)

\begin{abstract}
Проведены исследования влияния состава и температуры на электропроводность и диэлектрическую проницаемость системы $\mathrm{Tl}\left(\mathrm{GaS}_{2}\right)_{1-x}\left(\mathrm{InSe}_{2}\right)_{x}$. Установлено, что с ростом температуры диэлектрическая проницаемость и удельная проводимость увеличиваются, а с ростом концентрации $\mathrm{InSe}_{2}$ изменяются по линейному закону, при этом проводимость уменьшается, а диэлектрическая проницаемость растет. Подтверждено наличие в исследованной системе двух рядов твердых растворов. Показано, что с ростом концентрации $\mathrm{InSe}_{2}$ температуры фазовых переходов соразмерная-несоразмерная фаза смещаются в область более низких температур.
\end{abstract}

Работа выполнена при поддержке Белорусского фонда фундаментальных исследований (договор № Ф15-016 от 04 мая 2015 г.).

DOI: 10.21883/FTT.2017.08.44745.22

\section{1. Введение}

Кристаллы $\mathrm{TlGaS}_{2}$ и $\mathrm{TlInSe}_{2}$ относятся к большому семейству трехкомпонентных полупроводниковых соединений типа $\mathrm{Tl} B{ }^{\mathrm{III}} C_{2}^{\mathrm{VI}}(B=\mathrm{In}, \mathrm{Ga} ; C=\mathrm{S}, \mathrm{Se})$, которые представляют значительный научный и практический интерес. Одной из особенностей этого семейства является сильная анизотропия физических характеристик, обусловленная ярко выраженной слоистой кристаллической структурой. Поскольку взаимодействие между слоями в этих кристаллах осуществляется слабыми вандер-ваальсовыми силами, слоистые пакеты могут легко сдвигаться относительно друг друга. Это приводит к политипизму у многих представителей семейства $\mathrm{T} 1 B{ }^{\mathrm{III}} C_{2}^{\mathrm{VI}}$, что существенным образом влияет на их физические свойства [1-3].

Следует отметить, что кристаллы этого семейства проявляют как полупроводниковые, так и сегнетоэлектрические свойства. Кроме того, у некоторых кристаллов этой группы обнаружены последовательности фазовых переходов (ФП), обусловленные наличием в них длиннопериодических соизмеримых и несоизмеримых сверхструктур и их преобразованием с изменением температуры [4-6].

В обзоре [7] приведен анализ результатов исследований физических свойств соединений группы $\mathrm{Tl} B{ }^{\mathrm{III}} C_{2}^{\mathrm{VI}}$. Показано, что, несмотря на многочисленные исследования этих соединений, многие их физические характеристики не совпадают, в частности, значения температур
ФП, приводимые разными авторами для одних и тех же кристаллов, сильно различаются. Такие расхождения обусловлены прежде всего тем, что модулированная сверхструктура очень чувствительна к состоянию образца. Структурные дефекты, различные примеси, наличие политипии и др. оказывают большое влияние на температуры ФП в этих кристаллах.

Следует отметить, что, несмотря на многочисленные публикации, посвященные исследованиям различных физических свойств кристаллов $\mathrm{TlGaS}_{2}$ и $\mathrm{TlInSe}_{2}$, прямых доказательств существования в них модулированных структур нет. Хотя в ряде работ [8-10], посвященных исследованию различных физических свойств данных соединений в зависимости от температуры, выявлены аномалии на кривых этих зависимостей, что свидетельствует о наличии ФП.

Поскольку соединения группы $\mathrm{T} 1 B^{\mathrm{III}} C_{2}^{\mathrm{VI}}$ оптически активны, обладают высокой фоточувствительностью в широком спектральном диапазоне и перспективны для применения в оптоэлектронике, значительный интерес представляют твердые растворы этих соединений. На их основе можно получать материалы с непрерывно изменяющимися свойствами в зависимости от состава, что дает возможность получать кристаллы с наиболее оптимальными характеристиками для практического применения. Поэтому целью настоящей работы является установление закономерностей изменения электрических и диэлектрических характеристик твердых растворов $\mathrm{Tl}\left(\mathrm{GaS}_{2}\right)_{1-x}\left(\mathrm{InSe}_{2}\right)_{x}$ в зависимости от состава и температуры. 


\section{2. Методика эксперимента}

Измерения диэлектрической проницаемости $\varepsilon$ и электропроводности $\sigma$ монокристаллических образцов $\mathrm{Tl}\left(\mathrm{GaS}_{2}\right)_{1-x}\left(\mathrm{InSe}_{2}\right)_{x}$ проводились методом плоского конденсатора на монокристаллических пластинках размером $\sim 2 \times 5 \times 1 \mathrm{~mm}$ с помощью цифрового измерителя E7-20 на частотах измерительного поля $10^{3}-10^{6} \mathrm{~Hz}$ в температурном диапазоне $150-320 \mathrm{~K}$. Измерения осуществлялись перпендикулярно естественным плоскостям скола: (001) для образцов с $x=0,0.2,0.4,0.7$ и $(110)$ для образцов с $x=0.9,1$. На образцы наносили омические серебряные контакты и помещали между металлическими прижимными контактами. Держатель с образцом экранировали латунным стаканом, на который через изоляционную прослойку из слюды наматывали нагреватель. Питание нагревателя осуществлялось постоянным током от стабилизированного источника. Температура контролировалась при помощи дифференциальной хромель-копелевой термопары и универсального цифрового вольтметра. Измерения проводились методом непрерывного квазистатического нагревания со скоростью $\sim 0.5 \mathrm{~K} / \mathrm{min}$. Точность измерения температуры составляла $0.1-0.2 \mathrm{~K}$. Погрешность измерений диэлектрических характеристик составляла $\sim 0.5 \%$. Значения диэлектрической проницаемости и удельной электропроводности рассчитывались по формуле, соответствующей плоскопараллельному конденсатору.

\section{3. Результаты исследований}

Проведенные нами ранее рентгенографические исследования [11] показали, что в системе $\mathrm{Tl}\left(\mathrm{GaS}_{2}\right)_{1-x}\left(\mathrm{InSe}_{2}\right)_{x}$ существуют два ряда твердых растворов: на основе соединения $\mathrm{TlGaS}_{2}$ с моноклинной структурой в области $\mathrm{TlGaS}_{2}-\mathrm{Tl}\left(\mathrm{GaS}_{2}\right)_{0.3}\left(\mathrm{InSe}_{2}\right)_{0.7}$ и $\mathrm{TlInSe}_{2}$ с тетрагональной структурой в области $\mathrm{TlInSe}_{2}-\mathrm{Tl}\left(\mathrm{GaS}_{2}\right)_{0.1}\left(\mathrm{InSe}_{2}\right)_{0.9}$. Представляло интерес провести исследования электрических и диэлектрических характеристик этой системы.

На рис. 1 представлены температурные зависимости диэлектрической проницаемости монокристалла $\mathrm{Tl}\left(\mathrm{GaS}_{2}\right)_{0.3}\left(\mathrm{InSe}_{2}\right)_{0.7}$ (a) и $\mathrm{Tl}\left(\mathrm{GaS}_{2}\right)_{0.1}\left(\mathrm{InSe}_{2}\right)_{0.9}$ (b), полученные на различных частотах измерительного поля. Как видно из рисунка, значения $\varepsilon$ с ростом температуры увеличиваются и наблюдается значительная дисперсия диэлектрической проницаемости. С ростом частоты значения $\varepsilon$ уменьшаются, что обусловлено, как известно, релаксационными процессами, происходящими в высокочастотной области измерений. На кривых $\varepsilon=f(T)$ монокристалла $\mathrm{Tl}\left(\mathrm{GaS}_{2}\right)_{0.3}\left(\mathrm{InSe}_{2}\right)_{0.7}$ (см. рис. $\left.1, a\right)$ в области температур $\sim 180-240 \mathrm{~K}$ наблюдается широкий максимум. Ранее при исследовании теплоемкости [12] и оптических свойств [9] кристаллов $\mathrm{TlGaS}_{2}$ было установлено, что в области температур $\sim 173 \mathrm{~K}$ и $\sim 238 \mathrm{~K}$ имеют место аномалии соответствующих физических
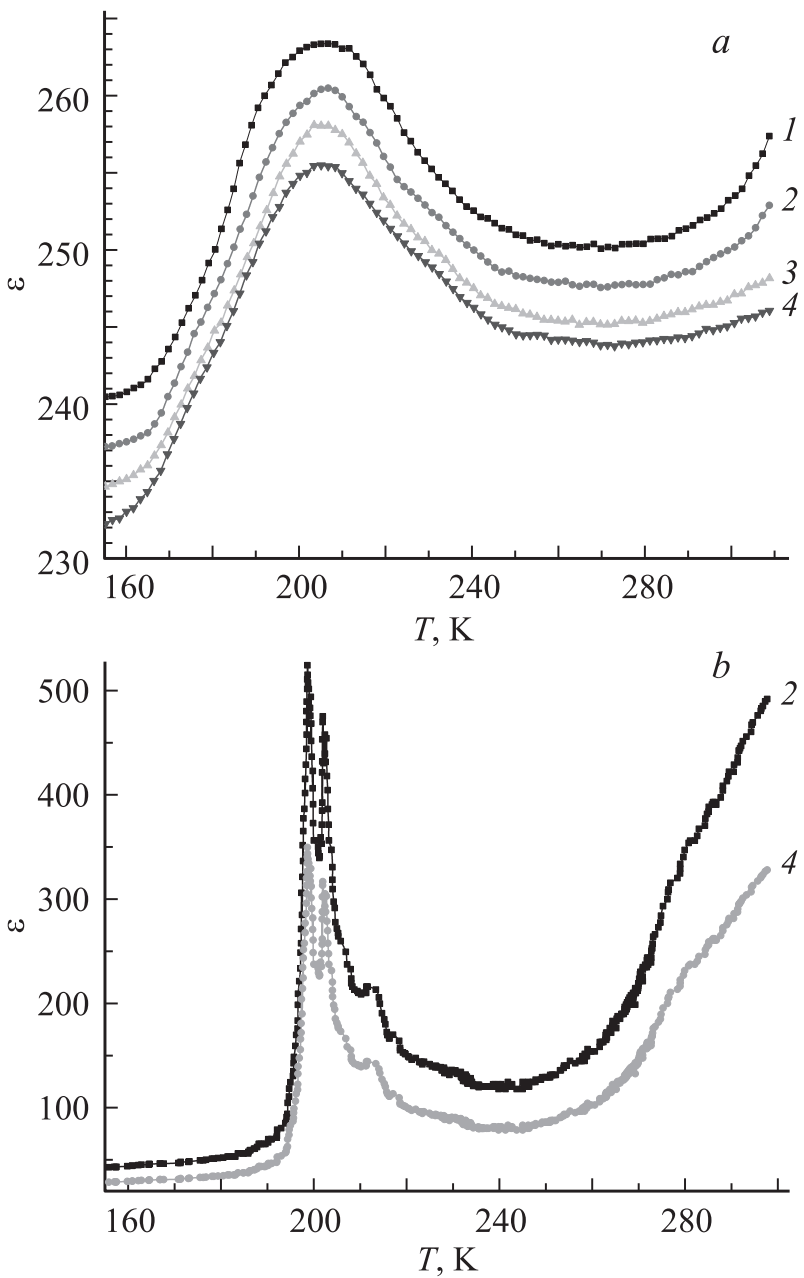

Рис. 1. Температурные зависимости диэлектрической проницаемости монокристалла $\mathrm{Tl}\left(\mathrm{GaS}_{2}\right)_{0.3}\left(\mathrm{InSe}_{2}\right)_{0.7} \quad$ (a) и $\mathrm{Tl}\left(\mathrm{GaS}_{2}\right)_{0.1}\left(\mathrm{InSe}_{2}\right)_{0.9}(b)$ на частотах $10^{3}(1), 10^{4}(2), 10^{5}(3)$ и $10^{6} \mathrm{~Hz}(4)$.

свойств. Возможно, в области температур 180-240 K существует несоизмеримая фаза, и поэтому наблюдается такой характер температурной зависимости $\varepsilon$. На кривых температурных зависимостей $\varepsilon=f(T)$ монокристалла $\mathrm{Tl}\left(\mathrm{GaS}_{2}\right)_{0.1}\left(\mathrm{InSe}_{2}\right)_{0.9}$ (см. рис. $\left.1, b\right)$ также обнаружены три аномалии: при $T_{i}=212 \mathrm{~K}, T_{c 1}=202 \mathrm{~K}$ и $T_{C 2}=198.8 \mathrm{~K}$, соответствующие фазовым переходам из парафазы в несоразмерную фазу $\left(T_{i}\right)$ и из несоразмерной фазы в соразмерную, причем происходит расщепление ФП при $T_{C}$ на два $\left(T_{C 1}\right.$ и $\left.T_{C 2}\right)$. Аналогичные результаты получены в [10] при исследовании диэлектрических свойств кристаллов $\mathrm{TlInSe}_{2}$.

На рис. 2 приведены температурные зависимости диэлектрической проницаемости $\mathrm{Tl}\left(\mathrm{GaS}_{2}\right)_{1-x}\left(\mathrm{InSe}_{2}\right)_{x}$ для разных $x$ со стороны $\mathrm{TlGaS}_{2}(a)$ и со стороны $\mathrm{TlInSe}_{2}(b)$. Температурные зависимости получены на частоте измерительного поля $1 \mathrm{MHz}$. Видно, что частичное замещение $\mathrm{TlGaS}_{2} \rightarrow \mathrm{TlInSe}_{2}$ приводит к увеличению значений диэлектрической проницаемости и уменьшению 

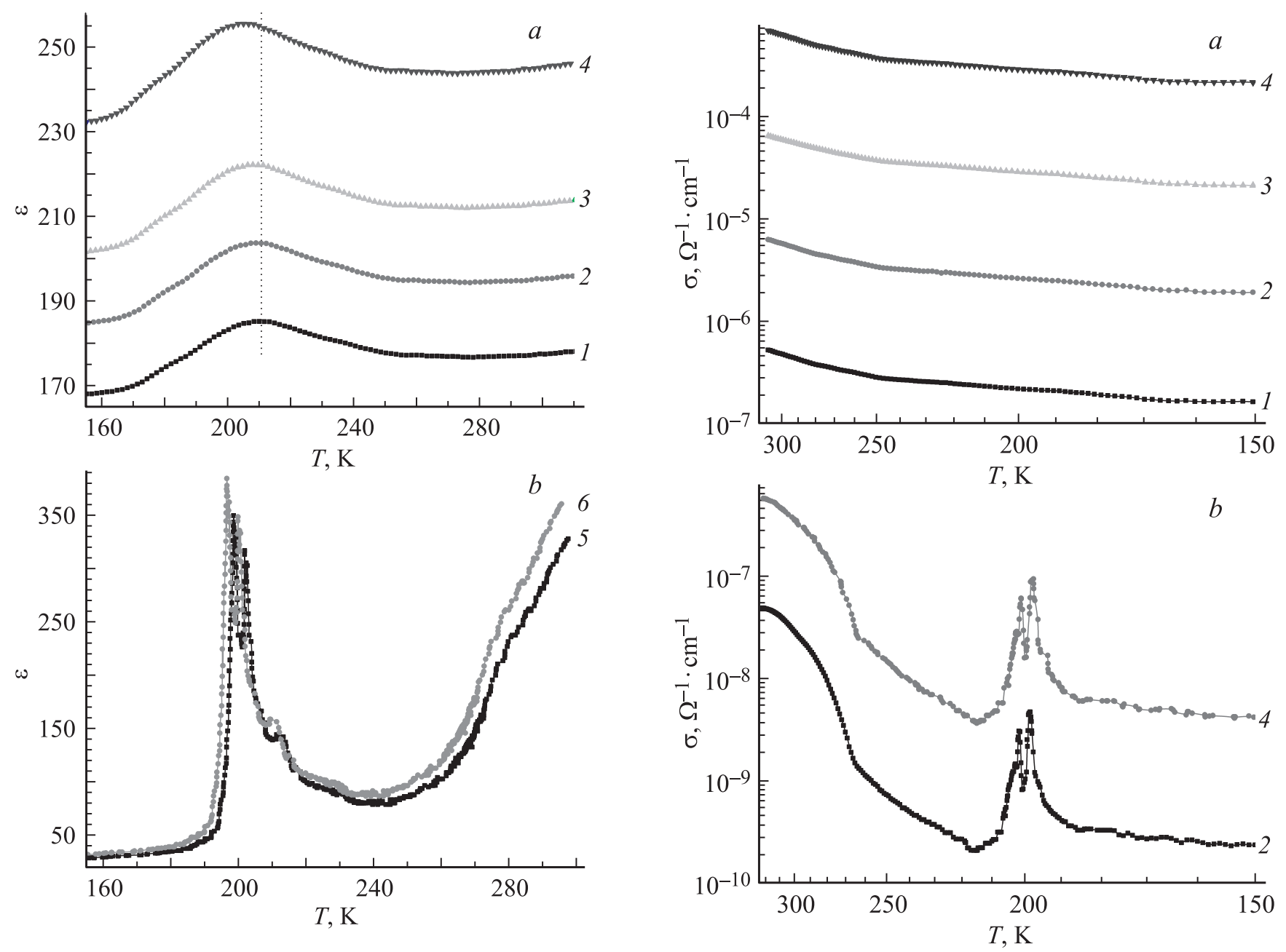

Рис. 2. Температурные зависимости диэлектрической проницаемости $\mathrm{Tl}\left(\mathrm{GaS}_{2}\right)_{1-x}\left(\mathrm{InSe}_{2}\right)_{x}$ со стороны $\mathrm{TlGaS}_{2}(a)$ для $x=0$ (1), 0.2 (2), 0.4 (3), 0.7 (4) и со стороны $\mathrm{TIInSe}_{2}(b)$ для $x=0.9(5), 1(6)$.

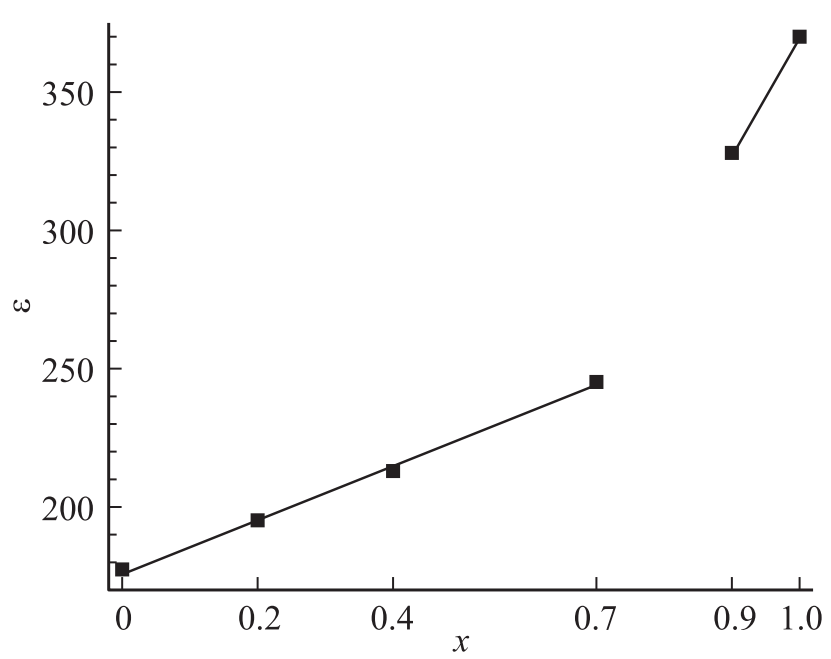

Рис. 3. Концентрационная зависимость диэлектрической проницаемости монокристаллов $\mathrm{Tl}\left(\mathrm{GaS}_{2}\right)_{1-x}\left(\mathrm{InSe}_{2}\right)_{x}$ на частоте $1 \mathrm{MHz}$ при температуре $300 \mathrm{~K}$.
Рис. 4. Температурные зависимости удельной электропроводности монокристалла $\mathrm{Tl}\left(\mathrm{GaS}_{2}\right)_{0.3}\left(\operatorname{InSe}_{2}\right)_{0.7}(a)$ и $\mathrm{Tl}\left(\mathrm{GaS}_{2}\right)_{0.1}\left(\mathrm{InSe}_{2}\right)_{0.9}\left(\right.$ b) на частотах $10^{3}(1), 10^{4}(2), 10^{5}(3)$, $10^{6} \mathrm{~Hz}(4)$.

температуры фазовых переходов. На рис. 3 представлена концентрационная зависимость диэлектрической проницаемости монокристаллов $\mathrm{Tl}\left(\mathrm{GaS}_{2}\right)_{1-x}\left(\mathrm{InSe}_{2}\right)_{x}$, полученная на частоте измерительного поля $1 \mathrm{MHz}$ при температуре $300 \mathrm{~K}$. Видно, что с увеличением концентрации $\mathrm{InSe} 2$ диэлектрическая проницаемость линейно возрастает, причем с разной скоростью для различных кристаллических структур твердых растворов.

На рис. 4 представлены температурные зависимости удельной электропроводности монокристалла $\mathrm{Tl}\left(\mathrm{GaS}_{2}\right)_{0.3}\left(\mathrm{InSe}_{2}\right)_{0.7}$ (a) и $\mathrm{Tl}\left(\mathrm{GaS}_{2}\right)_{0.1}\left(\mathrm{InSe}_{2}\right)_{0.9}(b)$, полученные на различных частотах измерительного поля. Из рисунка видно, что значения $\sigma$ увеличиваются с ростом температуры, что обусловлено ростом концентрации свободных носителей заряда (проявление полупроводниковых свойств). Участки с разным наклоном на кривых $\sigma=f(T)$ указывают на сложный механизм переноса заряда и наличие нескольких типов проводимости в этих кристаллах. Показано, что наблюдается значитель- 
ная дисперсия электропроводности. С ростом частоты значения $\sigma$ сильно увеличиваются, что обусловлено релаксационными процессами, происходящими в высокочастотной области измерений. На кривых $\sigma=f(T)$ также обнаружены аномалии, связанные с фазовыми переходами. Особенно ярко выражены они у твердых растворов на основе $\mathrm{TlInSe}_{2}$ (см. рис. $\left.4, b\right)$.

На рис. 5 приведены температурные зависимости удельной электропроводности $\mathrm{Tl}\left(\mathrm{GaS}_{2}\right)_{1-x}\left(\mathrm{InSe}_{2}\right)_{x}$, полученные на частоте измерительного поля $1 \mathrm{MHz}$. Как видно из рисунка, частичное замещение $\mathrm{TlGaS}_{2} \rightarrow \mathrm{TlInSe}_{2}$ приводит к уменьшению значений проводимости. Также обнаружено уменьшение температур фазовых переходов. На рис. 6 представлена концентрационная зависимость удельной электропроводности монокристаллов $\mathrm{Tl}\left(\mathrm{GaS}_{2}\right)_{1-x}\left(\mathrm{InSe}_{2}\right)_{x}$ при температуре $300 \mathrm{~K}$ на частоте измерительного поля $1 \mathrm{MHz}$. Видно, что в системе $\mathrm{Tl}\left(\mathrm{GaS}_{2}\right)_{1-x}\left(\mathrm{InSe}_{2}\right)_{x}$ с увеличением концентрации $\mathrm{InSe}_{2}$ проводимость линейно уменьшается. Изменение электропроводности на три

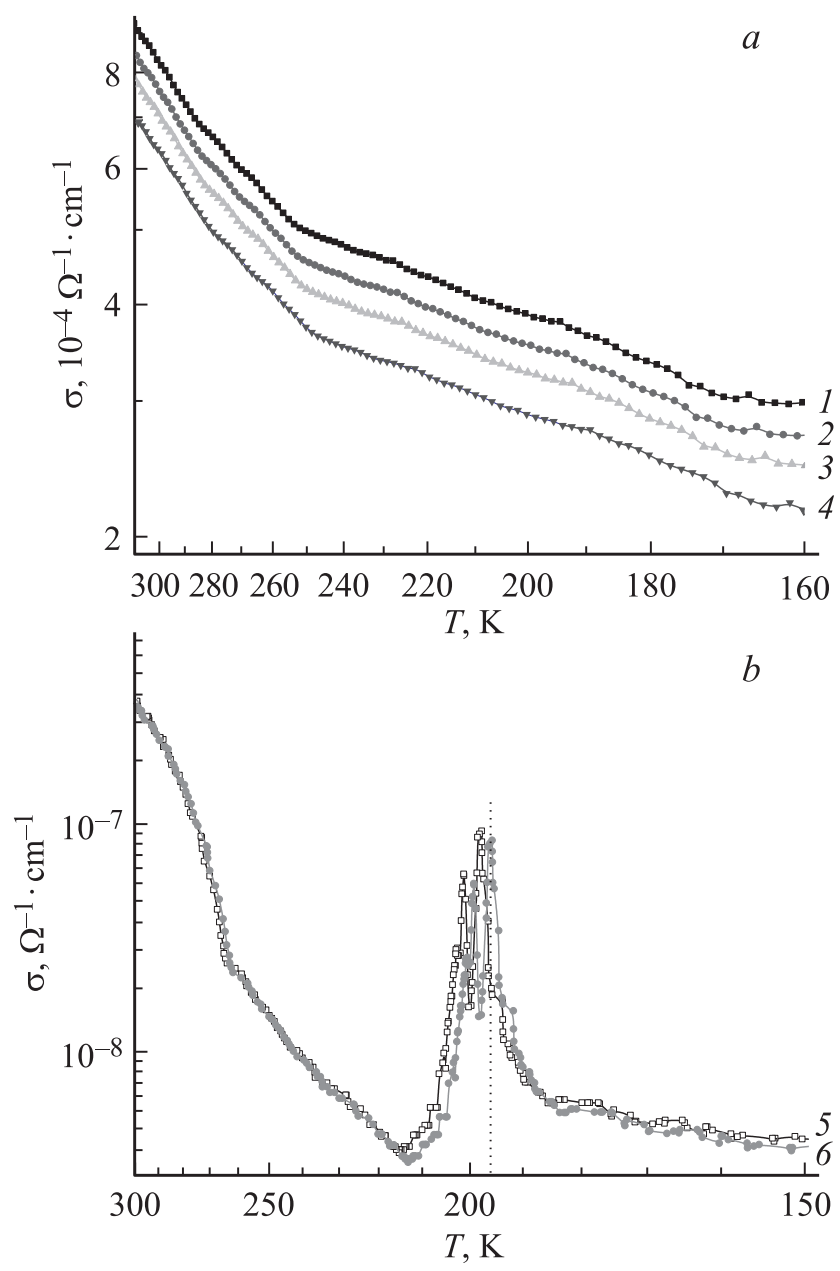

Рис. 5. Температурные зависимости удельной электропроводности $\mathrm{Tl}\left(\mathrm{GaS}_{2}\right)_{1-x}\left(\operatorname{InSe}_{2}\right)_{x}$ со стороны $\mathrm{TlGaS}_{2}(a)$ для $x=0(1)$, $0.2(2), 0.4(3), 0.7(4)$ и со стороны TlInSe $2(b)$ для $x=0.9(5)$, $1(6)$.

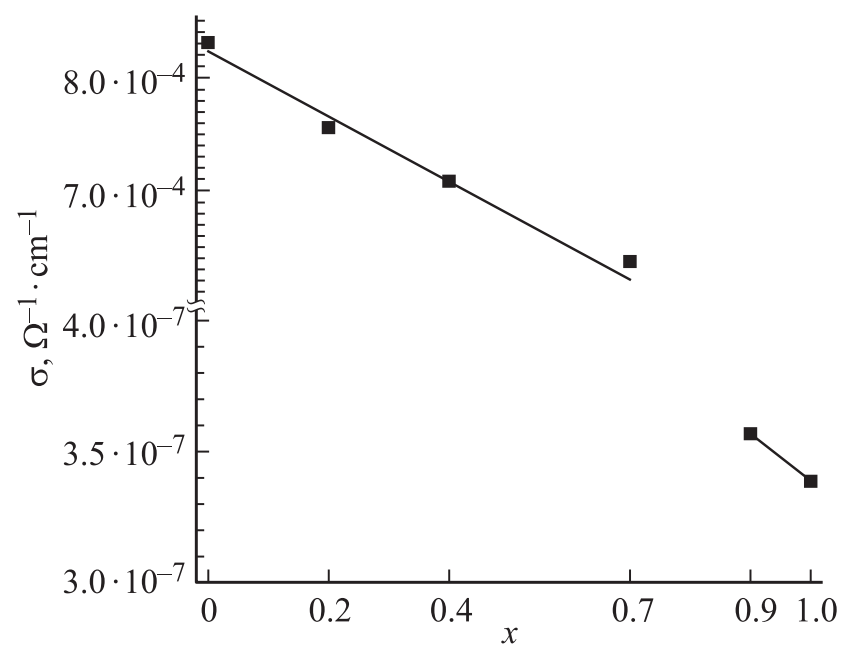

Рис. 6. Концентрационная зависимость удельной электропроводности монокристаллов $\mathrm{Tl}\left(\mathrm{GaS}_{2}\right)_{1-x}\left(\mathrm{InSe}_{2}\right)_{x}$ на частоте $1 \mathrm{MHz}$ при температуре $300 \mathrm{~K}$.

порядка при переходе от одного ряда твердых растворов к другому обусловлена тем, что измерения проводились вдоль различных кристаллографических направлений: [001] для образцов с $x=0,0.2,0.4,0.7$ и [110] для образцов с $x=0.9,1$. Из-за ярко выраженной слоистой структуры для кристаллов этого типа характерна сильная анизотропия физических свойств.

\section{4. Заключение}

Установлены закономерности изменения электропроводности и диэлектрической проницаемости системы $\mathrm{Tl}\left(\mathrm{GaS}_{2}\right)_{1-x}\left(\mathrm{InSe}_{2}\right)_{x}$ от состава и температуры. Показано, что с ростом температуры диэлектрическая проницаемость и удельная проводимость увеличиваются, а с ростом концентрации $x$ изменяются по линейному закону, при этом проводимость уменьшается, а диэлектрическая проницаемость растет.

Подтверждено наличие в системе $\mathrm{Tl}\left(\mathrm{GaS}_{2}\right)_{1-x}\left(\mathrm{InSe}_{2}\right)_{x}$ двух рядов твердых растворов: на основе соединения $\mathrm{TlGaS}_{2}$ с моноклинной структурой в области $\mathrm{TlGaS}_{2} \rightarrow \mathrm{Tl}\left(\mathrm{GaS}_{2}\right)_{0.3}\left(\mathrm{InSe}_{2}\right)_{0.7}$ и на основе соединения T1InSe ${ }_{2}$ с тетрагональной структурой в области $\mathrm{Tl}\left(\mathrm{GaS}_{2}\right)_{0.1}\left(\mathrm{InSe}_{2}\right)_{0.9}-\mathrm{TlInSe}_{2}$.

Показано, что в системе $\mathrm{Tl}\left(\mathrm{GaS}_{2}\right)_{1-x}\left(\operatorname{InSe}_{2}\right)_{x}$ с ростом концентрации $\mathrm{InSe}_{2}$ фазовые переходы соразмерная-несоразмерная фаза смещаются в область более низких температур.

\section{Список литературы}

[1] О.Б. Плющ, А.У. Шелег. Кристаллография 44, 5, 873 (1999).

[2] О.З. Алекперов, А.И. Наджафов. Неорган. материалы 45, 1, 9 (2009). 
[3] Н.А. Боровой, Ю.П. Гололобов, Г.Л. Исаенко, Н.Б. Степанищев. ФТТ 51, 11, 2229 (2009).

[4] А.У. Шелег, О.Б. Плющ, В.А. Алиев. ФТТ 36, 1, 226 (1994).

[5] D.F. McMorrow, R.A. Cowley, P.D. Hatton, J. Banys. J. Phys.: Condens. Matter 2, 16, 3699 (1990).

[6] S. Kashida, Y. Kobayashi. J. Korean Phys. Soc. 32, S40 (1998).

[7] A.M. Panich. J. Phys.: Condens. Matter 20, 29, 293202 (2008).

[8] A. Aydinli, R. Ellialtioğlu, K.R. Allakhverdiev, S. Ellialtioğlu, N.M. Gasanly. Solid State Commun. 88, 5, 387 (1993).

[9] A. Ateş, B. Gürbulak, M. Yildirim, S. Doğan, S. Duman, T. Yildirim, S. Tüzemen. Turk. J. Phys. 26, 2, 127 (2002).

[10] А.У. Шелег, В.Г. Гуртовой, С.Н. Мустафаева, Э.М. Керимова. ФТТ 53, 3, 443 (2011).

[11] А.У. Шелег, В.Г. Гуртовой, В.А. Чумак, С.Н. Мустафаева, Э.М. Керимова. Кристаллография 61, 4, 564 (2016).

[12] С.Г. Абдуллаева, А.М. Абдуллаев, К.К. Мамедов, Н.Г. Мамедов. ФТТ 26, 2, 618 (1984). 